\title{
Re: Pricop et al.: "Sublingual desmopressin is efficient and safe in the therapy of lithiasic renal colic"
}

\author{
Pouya Entezari $^{1} \cdot$ Hadi Mirfazaelian $^{1}$
}

Received: 27 February 2017 / Accepted: 17 March 2017 / Published online: 23 March 2017

(C) Springer Science+Business Media Dordrecht 2017

\section{Editor,}

We read the study conducted by Pricop et al. entitled "Sublingual desmopressin is efficient and safe in the therapy of lithiasic renal colic" and wish to offer some comments [1]. Authors have reached interesting outcomes, and we appreciate their efforts for sharing their results. Authors indicate that sublingual desmopressin can be as effective as nonsteroidal anti-inflammatory drugs (NSAIDs) in renal colic pain management. They also demonstrate an additive analgesic effect for the combination of sublingual desmopressin and NSIADs in pain reduction. According to previous studies, authors discussed in their article that the combination of intranasal desmopressin with NSAIDs or opioids does not add any benefit as an adjunct for pain management. For the first time in 2016, we conducted a meta-analysis on ten studies in order to compare the efficacy of intranasal desmopressin with NSAIDs and opioids in management of pain in nephrolithiasis [2]. We observed that intranasal desmopressin is less effective in pain reduction in comparison with both NSIADs and opioids. We also demonstrated that intranasal desmopressin does not provide any benefit in combination with NSAIDs for pain control. However, our obtained results indicated that intranasal desmopressin in combination with opioids significantly reduces need for rescue treatment in patients. Therefore, it was concluded that intranasal desmopressin could be used as an adjunctive treatment with opioids to improve pain management.
As authors note, the interesting difference between their obtained results and previous studies could reside in the administration rout of desmopressin (sublingual vs. intranasal). It is also noteworthy that besides the administration rout, Pricop et al. study was different from previous trials in using a higher dose of desmopressin (60 and $120 \mu \mathrm{g}$ vs. $40 \mu \mathrm{g}$ ) and different type of NSAID (ketorolac vs. diclofenac). Therefore, with these assumptions, the difference in results can be better explained.

\section{Compliance with ethical standards}

Conflict of interest None.

Ethical approval This article does not contain any studies with human participants performed by any of the authors.

\section{References}

1. Pricop C, Branisteanu DD, Orsolya M, Puia D, Matei A, Checherita IA (2016) Sublingual desmopressin is efficient and safe in the therapy of lithiasic renal colic. Int Urol Nephrol 48(2):183-189

2. Jalili M, Entezari P, Doosti-Irani A, Masoomi R, Mirfazaelian H (2016) Desmopressin effectiveness in renal colic pain management: systematic review and meta-analysis. Am J Emerg Med 34(8):1535-1541
Pouya Entezari

pouya_ent2003@yahoo.com

1 Department of Emergency Medicine, Tehran University of Medical Sciences, Tehran, Islamic Republic of Iran 